\title{
RTA Mathematic Simulation Principles at the Autotechnical Expertise
}

\author{
Badrudin Gasanov ${ }^{1 *}$, Artem Efimov ${ }^{1}$, and Jurij Grebennikov ${ }^{1}$ \\ ${ }^{1}$ Platov South-Russia State Polytechnic University (NPI), 132 Prosveschenia Str., Novocherkassk, \\ 346428, Russia
}

\begin{abstract}
The features of carrying out an autotechnical expertise (ATE) are considered in case the vehicles $(\mathrm{V})$ participating in the road transport accident (RTA) don't leave skid imprints. The examples of momentum and energy conservation law application are given at developing the road accident mathematical model. Special attention is paid to the determination methods of vehicle $(\mathrm{V})$ velocity, travel directions in various RTA diagrams and archeology of deformation. For this purpose it is offered to draw a momentum vector diagram. It is reasonable that for the calculation of $\mathrm{V}$ deformation at RTA it is necessary to determine step by step the strain-stress state in a contact area on the basis of the theories of elasticity, plasticity, solid friction and finite-element methods. The technique of constructing an RTA mathematical model is developed. It is recommended to use at ATE of RTAs at the runs-over into the fixed obstacle (a stationary V) and collisions.
\end{abstract}

\section{Introduction}

When carrying out an RTA ATE, investigating authorities most commonly put the following questions to the experts [1-3]:

- At which rate did the Vs move at the moment of emergency occurrence on the road and collision (run-over)?

- Did the drivers have technical capability to prevent RTA by emergency brake application or manipulation?

- Did (or didn't) the actions of RTA participants correspond to the requirements of traffic regulations and what discrepancies caused RTA?

As it is known [4-6], to answer the specified questions when carrying out ATE, the experts generally determine a stopping $\left(S_{0}\right)$ and a braking distance $\left(S_{T}\right)$ according to the following formulae:

$$
S_{\mathrm{o}}=\frac{V_{0}}{3,6}\left(t_{\mathrm{s}}+0,5 t_{\mathrm{n}}\right)+\frac{V_{0}^{2}}{26 j_{\text {ust }}} ; \quad S_{\mathrm{T}}=A V_{0}+\frac{V_{0}^{2}}{26 j_{\text {ust }}},
$$

where $V_{0}$ is a $V$ initial velocity, $\mathrm{km} / \mathrm{h}$;

$t_{s}, t_{n}$ are braking system delay and slowing-down rise time;

* Corresponding author: gasanov.bg@gmail.com 
$j_{\text {ust }}$ is a mean fully developed deceleration;

A is a coefficient characterizing braking action time [7].

In the formulae (1) the initial velocity is unknown. In the case of emergency brake application from the equations (1) we get [2-4]:

$$
\begin{gathered}
V_{0}=0,5 j t_{3}+\sqrt{2 j S_{\mathrm{u}}} ; \\
V_{0}=0,5 j t_{3}+\sqrt{2 j S_{\mathrm{u}}+V_{\mathrm{ps}}^{2}},
\end{gathered}
$$

where $j$ is a $V$ deceleration during braking force acceleration to the largest extremum $\left(t_{a}\right)$;

$S_{u}$ is a skid trace;

$V_{p s}$ is $V$ velocity at the moment of the first collision (run-over).

Over the past three years, the analysis of sales market in Russia shows that the vast majority of motor cars sold in the territory of Russia is equipped with antilock brake system (ABS) [8]. Considering that the $V$ with ABS at emergency brake application don't leave skid imprints virtually, the formulae (2) and (3) cannot be used to answer the raised questions. Therefore it is necessary to improve or develop new ATE methods, using laws of mechanics, the theory of ABS service properties, continuum mechanics and the theory of plasticity.

\section{Theory}

The objective of this work is generalization of mathematical models development possible options for RTA expertise allowing to answer the main questions arising at ATE.

According to the energy conservation law $[9,10]$ the change of $V$ kinetic energy (work) at RTA is equal to the equivalent force power $(F)$ in time. For example, at run-over into nondeforming obstacle

$$
\frac{d T}{d t}=\frac{\delta A}{d t}=F \frac{d h_{\mathrm{def}}}{d t}=F j_{3},
$$

where $h_{\text {def }}$ is a deformation depth;

$j_{3}$ is a $V$ deceleration degree.

All the forces operating on the $V$ at RTA can be divided into external and internal ones. Neglecting the work of $V$ contacting details inertia forces, we assume that the work of external forces is equal to the work of interaction forces. Potential and dissipative forces are referred to internal forces. Therefore, at $V$ collisions

$$
d T=\delta A_{\mathrm{wz}}=\delta A_{\mathrm{pot}}+\delta A_{\mathrm{dis}},
$$

where

$$
d T=d\left(\frac{m_{1} V_{1}^{2}}{2}\right)+d\left(\frac{m_{2} V_{2}^{2}}{2}\right)-d\left(\frac{m_{1} V_{1}^{\prime 2}}{2}\right)-d\left(\frac{m_{2} V_{2}^{\prime 2}}{2}\right)
$$

$V_{i}$ is $V$ velocity at a collision (run-over).

Potential forces incremental work is equal to the sum of elastic and plastic deformation incremental work:

$$
\delta A_{\mathrm{pot}}=\delta A_{\mathrm{upr}}+\delta A_{\mathrm{pl}} .
$$

It is possible to refer sliding friction and temperature change incremental work in the course of RTA to work of dissipative forces. Neglecting the last components, we receive 


$$
\delta_{A_{\mathrm{dis}}}=\delta_{A_{\mathrm{tr}}}=F_{\mathrm{tr}} d S_{\mathrm{\kappa}}=\mu_{\mathrm{\kappa}} P_{\mathrm{\kappa}} d S_{\mathrm{\kappa}},
$$

where $F_{t r}, \mu_{k}$ are solid friction force and coefficient;

$d S_{k}$ is a sliding path of each element in the Vs interaction zone;

$P_{k}$ is a correct pressure.

According to deformation mechanics all RTA can be conditionally divided into three types: perfectly elastic, perfectly inelastic (perfectly plastic solid) and elasto-plastic ones. It means that in the first case we neglect plastic (permanent) deformation of the $V$ at RTA. In the second case all kinetic energy of the $V$ at RTA is consumed for plastic deformation. The third option is the most reliable one when at RTA the work of deformation includes both plastic and elastic components. For the analytical description of these three options it is better to use momentum and energy conservation law.

Perfectly elastic collision, as a result of which internal energy of the $V$ (system) doesn't change. Respectively, kinetic energy of system doesn't change either. We will consider front central collision at which both the Vs move at different values of an impulse in the same straight line before and after the collision. Let us assume that before the collision the velocities of the $V s$ are equal to $V_{l}$ и $V_{2}$ in a rigid frame (Euler system) and the solids move towards each other or one of the $\mathrm{V}$ catches up with the other one (concurrent collision).

We will find an impulse of each body in the rigid frame ( $p 1$ and $p 2$ ). By means of the formulae velocities transformation at the transition from the relative system to the fixed one we will receive [9]:

$$
\begin{aligned}
& \boldsymbol{P}_{1}^{\prime}=m_{1} \boldsymbol{V}_{1}^{\prime}=m_{1}\left(\boldsymbol{V} \mathbf{c}+\boldsymbol{V}_{1}^{\prime}\right)=m_{1} \boldsymbol{V} \mathbf{c}+\tilde{\boldsymbol{P}}_{1}^{\prime} \\
& \boldsymbol{P}_{2}^{\prime}=m_{2} \boldsymbol{V}_{2}^{\prime}=m_{2}\left(\boldsymbol{V} \mathbf{c}+\boldsymbol{V}_{2}^{\prime}\right)=m_{2} \boldsymbol{V} \mathbf{c}+\tilde{\boldsymbol{P}}_{2}^{\prime}
\end{aligned}
$$

where $V_{c}$ is a presented mass axis velocity relative to the inspector system;

$\tilde{\boldsymbol{P}}_{i}^{\prime}$ is an impulse of $\mathrm{i}$ solid after the collision.

Inasmuch as, $\tilde{\boldsymbol{P}}_{1}^{\prime}=-\tilde{\boldsymbol{P}}_{2}^{\prime}$, so from the equations (9) and (10) we find

$$
\boldsymbol{P}_{1}^{\prime}+\boldsymbol{P}_{2}^{\prime}=\left(m_{1}+m_{2}\right) \boldsymbol{V} \mathrm{c}=\boldsymbol{P}_{1},
$$

further we will determine mass axis velocity vector projection from the formulae (9) и (10) on the axis $x$, coinciding with the direction of the $\mathrm{V}$ movement, according to the following formula:

$$
V_{c x}=\frac{m_{1} V_{1 x}+m_{2} V_{2 x}}{m_{1}+m_{2}}
$$

To determine the angle $\theta$ between the $V$ initial movement before and after the RTA (e.g., at the run-over into the stationary vehicle) the equation (11) will be demonstrated diagrammatically $[9,10]$. It is possible to establish the connection between the angles $\theta$ and $\theta_{1}$ :

$$
\operatorname{tg} \theta_{1}=\frac{\sin \theta}{\cos \theta+m_{1} / m_{2}} .
$$

Follows from the formula (12) that the angle of movement direction change after the $V$ collision with mass $m_{l}$ depends on the ratio of masses of both $V s$ and isn't defined unambiguously on the basis of momentum conservation law. But the vector diagram allows to define all possible directions of the movement of two $V s$ after the collision. However, if 
the $V s$ attitude after the collision and the RTA scene are fixed, then it is possible to estimate the direction of the $V$ movement before the RTA and their velocities.

Perfectly inelastic collision, as a result of which the collided Vs after the RTA move as a unit. In this case from the momentum conversation law (11) we have

$$
\left(m_{1}+m_{2}\right) \boldsymbol{V}^{\prime}=m_{1} \boldsymbol{V}_{1}+m_{2} \boldsymbol{V}_{2},
$$

where $V_{1}$ and $V_{2}$ are the velocities of the Vs with the masses $m_{1}$ and $m_{2}$ before the collision;

$V$ is their mass centre velocity after the collision.

In the central reference system before the collision both the $V s$ moved towards each other with the identical impulses $\tilde{\boldsymbol{P}}$, and after the collision the formed solid is motionless. Then total kinetic energy of solids entirely turns into internal energy, i.e. into the cumulative plastic deformation:

$$
\tilde{T}=\tilde{P}^{2} / 2 m_{1}+\tilde{P}^{2} / 2 m_{2}=\frac{m_{1} m_{2}}{2\left(m_{1}+m_{2}\right)} V_{o t n}^{2}=\frac{\tilde{P}^{2}}{2 M_{p r}},
$$

where $M_{p r}$ is the given mass of two $V s$.

From the equation (13) we find:

$$
T=A_{\text {pot }}=\frac{m_{1} m_{2}}{2\left(m_{1}+m_{2}\right)}\left(\boldsymbol{V}_{1}-\boldsymbol{V}_{2}\right)^{2}=\frac{M_{p r} V_{o t n}^{2}}{2} .
$$

Inelastic collision (elasto-plastic deformation of the objects of interaction at the RTA), as a result of which internal energy $\tilde{T}$ after the collision of one or both $V s$ changes as well as their movement direction (at least of the one of them). (14):

We will express the impulse of each $\mathrm{V}$ after the collision taking into account the formula

$$
\tilde{P}^{\prime}=\sqrt{2 M_{\mathrm{pr}}\left(\tilde{T}+A_{\mathrm{pot}}\right)} .
$$

For the definition of the possible Vs movement directions after the collision we will draw the vector diagram of the impulses.

We will show the option speed limit or kinetic energy estimation at which kinetic energy will be used only for deformation, i.e. for the increase in internal energy, and after the collision the interaction objects remain on the RTA scene or move as a single whole. In Laggrange reference system this assumption can be written down as follows:

$$
\tilde{T}_{\min }=A_{\text {pot }} .
$$

We will consider the case of run-over into the stationary $V$ when kinetic energy of one of the $V$ is close to zero. If after the collision both $V s$ didn't move, then their total impulse was equal to an impulse $p_{I}$ before the RTA of the first $V$, and kinetic energy of system

$$
T_{1}=\frac{P_{1}^{2}}{2\left(m_{1}+m_{2}\right)}=0 ; T_{1}=\delta A_{d} .
$$

If after the RTA both the $V s$ moved to a certain distance together, then the minimum kinetic energy of one of them, sufficient to their movement, was determined by the following formula 


$$
T_{1_{\text {min }}}=\left|A_{p o t}\right|+\frac{P_{1}^{2}}{2\left(m_{1}+m_{2}\right)}=R_{1} \frac{P_{1}^{2}}{2 m_{1}} ; \text { or } T_{1_{\text {min }}}=\frac{m_{1}+m_{2}}{m_{2}}\left|A_{p o t}\right|
$$

Whereas

$$
T_{1_{\min }}=\frac{m_{1} V_{1 \min }^{2}}{2} .
$$

so from the formulae (15) and (16) we find the speed limit of one of the $V$ before the collision above which they can move as a single whole, or, on the contrary, lower than which they remain on the RTA scene. For this purpose, we will determine $T_{\text {lmin }}$ by the following formula:

$$
T_{1_{\text {min }}}=\sqrt{\frac{2\left(m_{1}+m_{2}\right)\left|A_{p o t}\right|}{m_{1} m_{2}}} .
$$

In the formula (17) $\left|A_{\text {pot }}\right|$ is a system internal energy increment, that is the energy, consumed for the $V$ deformation work.

The incremental work of the $V$ design deformation at the RTA can be defined if their stress-strain state is known [11-13]:

$$
d A_{p}=\sigma_{i j} d \varepsilon_{i j}^{\mathrm{p}}+\sigma_{i j} \varepsilon_{i j}^{y},
$$

where $\sigma_{i j}$ are stress tensor components;

$\varepsilon_{i j}^{\mathrm{p}}, \varepsilon_{i j}^{\mathrm{y}}$ are the incremental plastic and elastic deformations of each particular element of the $\mathrm{V}$ in the process of RTA.

The connection between stress and deformation for an elastic solid is established on the basis of Hooke and Poisson's laws [Gun (1980), Ilyushin (1978)]:

$$
\begin{gathered}
\sigma_{i j}{ }^{-} \sigma_{s r} \delta_{i j}=2 G\left(\varepsilon_{i j}{ }^{-} \varepsilon_{s r} \delta_{i j}\right) ; \\
\varepsilon_{i j}^{\mathrm{y}}=\frac{1}{2 G}\left(d \delta_{i j}-\frac{3 v}{1+v} \delta_{i j} d \sigma_{\mathrm{sr}}\right),
\end{gathered}
$$

where $\sigma_{s r}, \varepsilon_{s r}$ are spherical tensor components:

$$
\begin{gathered}
\sigma_{s r}=\frac{1}{3}\left(\sigma_{11}+\sigma_{22}+\sigma_{33}\right) ; \\
\varepsilon_{s r}=\frac{1}{3}\left(\varepsilon_{11}+\varepsilon_{22}+\varepsilon_{33}\right) ;
\end{gathered}
$$

$\delta_{i j}$ is a Kronecker symbol.

For small elastic deformation [Gun (1980), Arkulis et al. (1987)]

$$
\varepsilon_{i j}=\frac{1}{2}\left(\frac{d U_{i}}{d_{x j}}+\frac{d U_{j}}{d_{x i}}\right),
$$

where $d U_{i}$ и $d U_{j}$ is distinguished element displacement vector change (e.g., a rectangle at the surface of deformed body element).

We will consider methods of incremental work definition of a body (cab) and other designs details plastic of the $V$. 
According to the deformation theory of plasticity [13-15] the incremental work of plastic deformation is proportional to an increment (change) of material volume and product form:

$$
d A_{p}=\sigma_{i j} d \varepsilon_{i j}=\sigma_{\mathrm{sI}} d \theta+\mathrm{E}=\mathrm{T} d \mathrm{G},
$$

where $T$ is a voltage deviator (tangent stress intensity);

$G$ is deformation shift intensity.

If at RTA fractions and infractions are not formed, then it is possible to neglect volume change at plastic deformation (as a result of flaw density increase) $d \theta$. Then

$$
d A_{p}=\mathrm{T} d \mathrm{G} .
$$

If the deformation tensor components $\varepsilon_{i j}$ are known, then $G$ is determined according to the following formula:

$$
G=\sqrt{\frac{2}{3}} \sqrt{\left(\varepsilon_{11}-\varepsilon_{22}\right)^{2}+\left(\varepsilon_{22}-\varepsilon_{33}\right)^{2}+\left(\varepsilon_{33}-\varepsilon_{11}\right)^{2}+\frac{2}{3}\left(\varepsilon_{12}^{2}+\varepsilon_{13}^{2}+\varepsilon_{23}^{2}\right)} .
$$

We cannot define plastic deformation work by formula integration (20), as far as $T$ depends on $G$. Consequently, it is necessary to employ supplementary conditions or various plasticity theories. In particular, if voltage deviator $T=\tau_{s}, \tau_{\mathrm{s}}=\delta_{s} / \sqrt{3}$ is equal to material yield strength (of steel, aluminum alloys), then instead of the equation (20) we can employ Hencky`s formula [13-15]:

$$
\varepsilon_{i j}=\frac{G}{2 \tau_{\mathrm{s}}} S_{i j}
$$

where $S_{i j}$ are deviator voltage tensor components characterizing shape change (configuration) of the detail element under investigation,

$$
S_{i j}=\sigma_{i j}{ }^{-} \sigma_{\mathrm{sr}} \delta_{i j} .
$$

It should be noted that the equation (18) isn't biunique, i.e. if the values $\varepsilon_{i j}$ are known, then it is possible to find the values $S_{i j}$, since the deformation deviator is also determined by the formula (22). If $S_{i j}$ are set, then it isn't possible to find $\varepsilon_{i j}$ as the deformation deviator $\mathrm{G}$ is unknown.

In irreversible processes of the V deformation at RTA it is very difficult to estimate the deformed condition, it is even more difficult to define strenuous condition, respectively deformation work too. In the theory of chipless process it is established that the voltage deviation is proportional to a deformation velocity deviator.

For the definition of $S_{i j}$ by the formulae (22) and (23) it is necessary to use the procedure of stage-by-stage deformation zone final geometric pattern at RTA. Various methods of such tasks solution are developed. The final element analysis has received the greatest application (FEA) [16-20]. The considered volumes (surfaces) are divided into n-elements of the set configuration. For example, at assessment of the deformed condition of a product from sheet material (steel, aluminum alloys, thermosetting plastic), we can present its surface as a combination of rectangles or triangles. In the moving coordinate system, the movement of each mount can be set by vectors $d U_{x y}$ and $d U y x$ or by velocities of movement $d V_{x y}$ and $d V y x$ respectively:

$$
\varepsilon_{x x}=\frac{d U_{x}}{d_{x}} ; \varepsilon_{y y}=\frac{d U_{y}}{d_{y}} ; \varepsilon_{y x}=\frac{1}{2}\left(\frac{\Delta U_{x}}{\Delta_{y}}+\frac{\Delta U_{y}}{\Delta_{x}}\right), \text { or }
$$




$$
\eta_{x x}=\frac{d V_{x}}{d_{x}} ; \eta_{y y}=\frac{d V_{y}}{d_{y}} ; \eta_{y x}=\frac{1}{2}\left(\frac{\Delta V_{x}}{\Delta_{y}}+\frac{\Delta V_{y}}{\Delta_{x}}\right) .
$$

In case of the sliding blow at RTA, i.e. when changes of kinetic energy are connected with the incremental work of contact sliding friction forces, it is necessary to consider that in the formula (4) the coefficient of a contact sliding friction considerably differs from a sliding friction in kinematic couples. It can change ranging from $0,2-0,5$, depending on the sliding velocity, a condition of a contact surface, material composition and character of external forces [15]. At the same time contact pressure $\left(P_{k}\right)$ can be determined if strenuous condition in a zone of deformation and contact surface total area are known.

\section{Conclusions}

It is reasonable that for ATE carrying out at collisions and runs-over it is necessary to comprise the closed equations called mathematical model and including: formulae for braking path definition taking into account road conditions; energy and impulses conservation laws; analytical dependences for assessment of the stress-strain state on the basis of finite element methods, the plasticity deformation theory or the developed plastic deformation; impact mechanics and dynamics and others [20].

The expert, who is entrusted to carry out ATE, is to prove all the assumptions made by him for simplification of mathematical model and to show the importance or insignificance of all the factors influencing results of expertise. There is also the option to include a subheading within the Appendix if you wish.

\section{References}

1. V.D. Balakin, Road traffic accident expertise: study guide. Omsk: SibADI, 136. (2010)

2. S.A. Evtyukov, Ya.V. Vasiliev Road traffic accidents expertise. Reference book - St. Petersburg.: Publishing office DNK, 536 pp. (2006)

3. V.A. Puchkin, Road traffic accident expert analysis fundamentals: Database. Expert technics. Solution methods. - Rostov-on-Don: IPO PI SFU,. - 400 pp. (2010)

4. V.I. Lozovoy, Road traffic accident investigation and expertise: study guide / V.I. Lozovoy, V.G. Tamadaev, A.D. Efimov; Platov SRSPU (NPI). - Novocherkassk: Publishing office SRSPU (NPI), 298 pp. (2015)

5. R. Byatt, Manual of road accident investigation: translated from English / R. Byatt, R. Watts. - M.: Transport, 288 pp. (1983)

6. V.A. Ilarionov, Road traffic accident expertise: Higher education institution textbook / V.A. Ilarionov. - M.: Transport, 255 pp. (1989)

7. All-Union State Standard R 51709-2001. Vehicles. Technical state safety specification and examination methods (edited from 28.03.2006) (2006)

8. Avtostat info [Electronic resource]. Access mode http://avtostat-info.com/ (2017)

9. N.A. Kiljchevskiy, Theoretical mechanics course. Vol. 1. Study guide. 2th edition. M.: Nauka, 1977. 480 pp. (1977)

10. N.A. Kiljchevskiy, Theoretical mechanics course. Vol. 2. Study guide. - M.: Nauka, 544 pp. (1977)

11. G.Ya. Gun, Metal working process theoretical foundations. (Plasticity theory), Higher education institution textbook. M., «Metallurgy», 456 pp. (1980) 
12. W.J ohnson, P.B. Mellor, Engineering Plasticity. translated from English / translated by A.G. Ovchinnikov. - M.: Mashinostroenie, 567 pp. (1979)

13. G.E. Arkulis, V.G. Dorogobid, Plasticity. Higher education institution textbook. M.: Metallurgy, 352 pp. (1987)

14. A.A. Ilyushin, Continuum mechanics. M.: Publishing office MSU, 287 pp. (1978)

15. M.V. Storozhev, E.A. Popov, Metal working process theory. M.: Mashinostroenie, 423 pp. (1977)

16. O. Zenkevich, The finite element method. Translated from English. - M.: Mir, 543 pp. (1975)

17. L. Segerlind, Applied finite element analysis. Translated from English/Translated by A.A. Shestakova. M.: Mir, 392 pp. (1979)

18. V.N. Nikonov, RTA mathematic models and their admissibility in a judicial process. // Vestnik Justitia. №1. P.15-20. (2007)

19. V.N. Nikonov, RTA mathematic model classification and their admissibility in a judicial process. // Legitimacy. №5. P.30-34. (2007)

20. E.F. Novopisniy, Vehicle bumper stress-strain state analysis by the method of finite elements / A.I. Shutov, E.A. Novopisniy // Transport information certificate. № 12. P. 20-23. (2011) 